\title{
Filling the void for new Alzheimer's disease therapy
}

\author{
The recent approval of a new drug for Alzheimer's disease despite weak evidence of efficacy sent shockwaves \\ throughout the scientific community. The approval leaves many open questions in its trail that must now \\ be addressed.
}

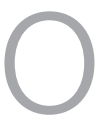
n 7 June, the US Federal Drug Administration (FDA) approved aducanumab, an anti-amyloid therapy for Alzheimer's disease (AD) developed by Biogen. As the drug is the first therapy approved for the disease in over 18 years, patients have welcomed the news enthusiastically. In contrast, the decision has polarized the research and clinical communities, leaving many puzzled as to why the drug was approved despite uncertainty on its clinical efficacy.

Unlike previously approved drugs that counter cognitive symptoms, aducanumab targets the biology of the disease, the amyloid- $\beta(\mathrm{A} \beta)$ plaques that accumulate in the brain of patients, a hallmark of AD. The trial data convincingly showed that aducanumab decreased $\mathrm{A} \beta$ deposition in the brain, but only one of the two Biogen trials suggested this was also associated with a slowing of cognitive decline as the disease progresses. Unexpectedly, the FDA did not follow the near unanimous recommendation (Nat. Aging 1, 324-326; 2021) of its own external advisory committee not to approve the drug based on the lack of statistical support for efficacy. Three advisory committee members resigned in protest.

Typically, the FDA requires two independent trials demonstrating efficacy to approve a new drug. These guidelines are not always followed, in particular for serious medical conditions with an important unmet need for a drug. Acknowledging the lack of support for clinical efficacy, the FDA granted aducanumab accelerated approval, a form of approval that can rely on a surrogate endpoint that is "reasonably likely to predict clinical benefit"; in this case, the removal of $A \beta$ from the brain. But it is difficult to see the logic behind this rationale given that to date, there is no definitive clinical evidence that plaque removal slows cognitive decline in $\mathrm{AD}$. In fact, several other drugs targeting A $\beta$ previously failed in clinical trials. To many experts, the decision appeared to rely more on belief than sound scientific evidence.
The aducanumab decision sets a precedent for a new approval standard for $\mathrm{AD}$ drugs that modify the disease by targeting $A \beta$ : one that relies on unconfirmed biomarker-based proxies of outcomes. As recent news indicates, this has opened the gate for accelerated approval filings based on this new standard.

As a condition for continued authorization to market a therapeutic product, an accelerated approval requires definitive demonstration of efficacy in a post-approval trial. In their approval letter to Biogen, the FDA gave the drugmaker a generous timeframe of nine years to complete this additional trial. The outcome of this yet-to-be-defined trial is of utmost importance given the existing doubts on clinical efficacy. It is therefore crucial that the FDA holds Biogen to its deadlines and, together with the company, ensures the new trial is adequately designed to provide an unequivocal answer to the efficacy question.

Uncertainty remains on the drug's efficacy but neurologists will soon start prescribing it, and will face several other uncertainties. The indication on the drug label is surprisingly broad, merely stating it is indicated for the "treatment of Alzheimer's disease". This broad indication ignores the fact that $\mathrm{AD}$ has several stages and that the patient population in which the drug was tested was only composed of patients with early-stage AD. Accumulation of $A \beta$ starts early in the disease, and many researchers think $A \beta$ may play a lesser role in late-stage $\mathrm{AD}$. A related and notable absence from the label's recommendations is the verification that patients indeed have plaques in their brain. $\mathrm{AD}$ is often misdiagnosed when based solely on cognitive and functional assessments, and a positron emission tomography (PET) scan to detect plaques is considered a diagnostic gold standard. Yet, there are no provisions for diagnosis in the label. Trying to remove plaques that do not exist would be senseless and administrating to late-stage $\mathrm{AD}$ patients could be futile. Even worse, either could potentially cause unnecessary harm, since the drug is not without side effects.
The trials revealed that about one-third of patients treated with the drug experienced adverse events called amyloid-related imaging abnormalities (ARIA), a form of brain swelling that can lead to brain hemorrhaging. Many older adults take anticoagulant medications for heart conditions, treatments that would likely increase the risk of severe complications in the event that ARIA occurs, and accordingly use of blood thinners was part of the exclusion criteria for the trials. Although the label warns about ARIA and makes specific recommendations for its monitoring, it does not mention any of the trials' exclusion criteria or feature any contraindications. Here again, the FDA appears to have left a void for clinicians to fill.

These gaps point to the urgent need for a more careful set of clinical care guidelines that establishes a consensus on who the drug can be prescribed to.

Aducanumab also comes with a steep price. The treatment itself will cost a whopping US\$56,000 per year, which does not include the expensive monitoring exams (magnetic resonance imaging (MRI) scans) that are necessary to ensure that the treatment is well tolerated. As six million Americans are currently diagnosed with $\mathrm{AD}$ and most are over the age of 65 , the high price tag has the potential to bankrupt the federal health insurance system in the USA and leave many patients with exorbitant out-of-pocket expenses. If nothing changes, this will also have an impact on access to treatment and likely lead to further health inequalities in AD. The nature of the treatment's coverage has yet to be determined by the Centre for Medicare and Medicaid Services (CMS), but legislators are already calling for CMS to consider a national coverage determination (NCD) that could restrict eligibility, and for Congress to consider policy reforms that would limit the cost of a drug based on its expected benefits. CMS, in consultation with leading dementia experts, should seize this opportunity to address some of the inadequacies that were left behind by the FDA. 
The development of aducanumab has been controversial and divisive, and its future appears fraught with difficulties. Close collaboration between regulators, clinicians and Biogen will be key to addressing the many challenges ahead until a definitive answer emerges on whether this new treatment works. In the meantime, doctors and their patients will remain in the unenviable situation of deciding whether to take substantial risks for gains that have yet to be confirmed.

Published online: 1 July 2021

https://doi.org/10.1038/s43587-021-00092-w 